\title{
3 Research Square

\section{Remote Patient Monitoring and classifying Using the Internet of Things Platform Combined with Cloud Computing}

Somayeh Iranpak

Islamic Azad University Sabzevar Branch

Asadollah Shahbahrami ( $\nabla$ shahbahrami@guilan.ac.ir)

University of Guilan https://orcid.org/0000-0002-5195-1688

Hassan Shakeri

Islamic Azad University Mashhad Branch

\section{Research}

Keywords: Internet of Things (IoT), cloud computing, remote patient monitoring, Patient data analysis

Posted Date: April 20th, 2021

DOI: https://doi.org/10.21203/rs.3.rs-430091/v1

License: (c) (i) This work is licensed under a Creative Commons Attribution 4.0 International License.

Read Full License 


\section{Remote Patient Monitoring and classifying Using the Internet of Things Platform Combined with Cloud Computing}

\section{Somayeh Iranpak ${ }^{a}$, Asadollah Shahbahrami ${ }^{b^{*}, \text { Hassan Shakeri }^{\mathrm{c}}}$}

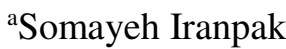

Ph.D. Candidate of Information Technology Engineering, Department of Computer

Engineering, Sabzevar Branch, Islamic Azad University, Sabzevar, Iran.

somayeh.iranpak@iaus.ac.ir, +989113368647

$b^{*}$ Asadollah Shahbahrami

Associate Professor of Computer Engineering, Department of Computer Engineering, Faculty of Engineering, University of Guilan, Rasht, Iran. Corresponding Author:

shahbahrami@guilan.ac.ir, +989118055726

${ }^{\mathrm{c}}$ Hassan Shakeri

Associate Professor of Computer Engineering, Department of Computer Engineering, Mashhad Branch, Islamic Azad University, Mashhad, Iran.

shakeri@mshdiau.ac.ir ,+989155138798 


\title{
Remote Patient Monitoring and classifying Using the Internet of Things Platform (IoT) Combined with Cloud Computing
}

\begin{abstract}
Patients' health and providing an optimal and appropriate solution have recently been considered by many researchers. With the advent of technologies such as cloud computing and 5G network, information can be exchanged faster and more securely. Using cloud computing in this process can significantly improve the monitoring of certain patients. Therefore, providing a favorable method in the medical industry and computer science to monitor the status of patients using connected sensors is very important. Thus, due to its optimal efficiency, speed, and accuracy of data processing and classification, the use of cloud computing to process the data collected from remote patient sensors and the Internet of Things (IoT) platform has been suggested. In this paper, a prioritization system is used to prioritize sensitive information in IoT, and in cloud computing, LSTM deep neural network is used to classify and monitor patients' condition remotely, which can be considered as an important innovative aspect of this paper. Sensor data in the IoT platform is sent to the cloud with the help of the 5th generation Internet. The core of cloud computing uses the LSTM deep neural network algorithm. Through simulating the proposed method and comparing the obtained results with other methods, it was observed that the accuracy of the proposed method has been improved significantly compared to other methods.
\end{abstract}

Keywords: Internet of Things (IoT), cloud computing, remote patient monitoring, Patient data analysis.

\section{Introduction}

Cloud computing is one of the most valuable and promising research paths [1]. This calculation method provides infrastructure and software services for users and provides services requested by users through the Internet. Considering the notable growth of cloud computing, the number of clients and the number of requests is increasing rapidly. Therefore, increasing the speed and accuracy of cloud computing is very important [2]. 
Microservice development is preferred to the development of integrated applications because applications with such an architecture offer the desired features but achieve greater flexibility, reliability, and scalability [3].

In recent years, the healthcare service sector has become one of the main targets of the academic community and the financial market. This sector has attracted a lot of funds, mostly because it has research potential. The healthcare sector faces many problems, including insufficient resources, high cost of healthcare, and inefficient management of hospital resources. There are also a number of logistics challenges, such as the amount of medical care that patients need, the limited number of beds, the limited equipment, and the small number of healthcare specialists. Patient monitoring is usually performed passively, meaning that medical teams administer medication in response to changes in patients' health status [4].

The response time is very important because in critical cases the patient's condition may worsen. The Internet of Things offers many opportunities in the field of e-health. This technology can improve health services and lead to various innovations in this regard [5]. The Internet of Things acts as a catalyst for healthcare and plays an important role in healthcare services [6]. Body sensor network (BSN) detects abnormal and unforeseen conditions by evaluating physiological parameters and symptoms and send vital signs for medical evaluation. Therefore, temporary medications can be prescribed immediately to prevent critical conditions. With the help of the Internet of Things, medical care processes are facilitated and through using smart systems, health services are also improved [7]. The Internet of Things provides a platform for achieving goals such as improving the quality of human life in terms of health and facilitating the daily lives of people with disabilities and chronic diseases. It has made life easier, especially for those who cannot be physically present to care for the patients [8]. 
The field of e-health [9] is a field of research that can have many benefits for society if it is properly employed. Some of the services that can be provided by the Internet of Things in the healthcare field include:

A) Automatic collection of data related to the patient's vital signs through a network of sensors connected to medical equipment and the patient's body (software agents constantly monitor the collected data to identify any abnormalities in the health status of patients and inform healthcare specialists.

B) Sending the collected data to the cloud platform of the medical center to be stored and processed.

C) Comprehensive analysis of mobile health plans and sharing medical data.

Besides, it allows remote storage of data on cloud-based platforms and enables the cooperation of any authorized person, including external specialists and the internal team [5]. It is also an appropriate platform for health applications based on mobile / tablet / PDA devices. The use of smartphones provides numerous opportunities to create effective healthcare services and solutions. This paper, under the title of improving the efficiency in remote patient monitoring using the Internet of Things and cloud computing, presents the simulation of a remote patient monitoring system based on the Internet of Things and cloud computing in two static (smart homes) and dynamic (mobile health) environments to increase the effectiveness of health services (in any place and any time) and improve the healthcare system using MATLAB simulator.

Therefore, the LSTM deep neural network is used in this paper to remotely classify and monitor the condition of patients. Data and information about the patients' condition are provided via sensors in the IoT and 5th generation Internet to cloud computing and as an input to the LSTM deep neural network. The LSTM Deep Neural Network is based on 
trained input data and produces a model based on hidden layers and neural structure. Then it receives information about patients 'status for classification and evaluation and uses the generated model to classify and monitor patients' health status remotely. In the following section, studies done in this field are reviewed. Section three deals with the proposed method. Finally, in sections 4 and 5, the obtained results will be presented, followed by the conclusion and suggestions for further studies.

\section{Related work}

In this section, research conducted in the field of monitoring patients' health status is reviewed, and then, papers published in recent years will be compared to each other. Table 1 This table outlines the advantages and disadvantages of each method.

In [4] a new platform is proposed for the smart home to analyze IoT big data with cloud and fog computing technology. The use of this model yielded good practical results for identifying problems and planning to save energy, maintenance, and so on.

In [5], a Remote Patient Monitoring System (RPMS) aims to effectively manage hospital resources through patient monitoring at home. RPMS is an IoT-based solution for patient monitoring that supports the automatic collection and transmission of patient data to remote databases. A web system provides access to this data through a userfriendly interface.

In [10] an innovative IoT-based system is developed for medication identification and prescription medication monitoring which is called Pharmaceutical intelligent information system (PIIS). This system can be used for examining medications to identify dangerous side effects, renal absorption reactions, side effects during pregnancy or lactation, and certain diseases such as tuberculosis. 
In [11] a home monitoring and decision support system is provided to assist physicians in diagnosis, remote monitoring, treatment, prescription, rehabilitation, and progression of patients with Parkinson's disease. This system is an expert system that collects tremor data and helps physicians diagnose and treat it.

In [12] an IoT-based health monitoring system is presented to investigate the role of smart data in the smart home considering remote health monitoring. Remote health monitoring is a technology that makes it possible to monitor the patients outside of clinical settings (usually at home), which can increase access to care and reduce the cost of providing healthcare.

In [13] an IoT-based mobile gateway (e.g. mobile / tablet / PDA, etc.) is developed for mobile health scenarios. The gateway automatically collects information such as location, heartbeat rate, and possible diagnosis, and promptly sends it to the caregivers.

In [14] an IoT-based health monitoring system is provided for children with autism. Health information for autistic patients was collected using a wearable sensor that is put on the head. Then the information read from the brains of these patients is continuously sent to the monitoring server. With any discrepancies in the information read, an alert is generated and an email is sent to the caregiver. In case of any emergency, alerts are also sent to the physician. Patient personal details can also be stored in a cloud environment for scalability and effective security.

In [15] an IoT-based framework for healthcare (HealthIoT) is provided in which ECGs and other healthcare data are collected by mobile devices and sensors and securely sent to the cloud so that the specialists have access to them. Signal enhancement techniques, encryption, and other related analyses have been used to prevent identity theft or clinical error. 
In [16] a system called Smart Architecture for In-Home Healthcare (SAHHC) is proposed, which is a healthcare solution using the image and facial expressions used to monitor patients and the elderly who have special needs.

In [17] an IoT-based monitoring framework in the Intensive Care Unit (ICU) is proposed to improve the delivery of medical services. The framework presented in this paper focuses on the efficient monitoring of various events (and anomalies) with temporary dependencies, followed by time-sensitive alerts. The results show that IoTequipped ICUs are much more efficient in monitoring sensitive events than manual and conventional Tele-ICU monitoring. Besides, the method adopted to generate alerts provides more information and increases the advantages of the system.

In [18] a system for reminding and monitoring medication use is proposed which works with the help of the Internet of Things. This paper focuses on the medication and its use schedule which is useful for improving the efficiency of the prescribed medication and reducing the economic factor. They observed some monitoring technologies that build the in-home monitoring system. The monitoring system can be implemented with sensor components and a wireless module that should be secured so that the message containing the health information is not distorted. The Internet of Things (IoT) plays a vital role in the communication between the two devices, the use of standard messages, and communication protocol so that we can securely communicate important health-related messages. The IoT open source cloud is efficient for storing sensor data. The advantage of digital storage is that it is easy to recover and performs faster in case of an emergency. RFID is the best option for the user's identity and encrypting/decrypting purposes.

[19] examines the IoT-based patient monitoring system. An IoT-based patient monitoring system is an alternative used to help patients with chronic diseases. The goal of this method is to improve the quality of life of patients-not only are they monitored, 
but they also gain the ability to improve their eating habits and exercise routine. The results of this study show that the developed model for the system at the time of making inferences and achieving results is as effective as orders to obtain measurements through sensors, as well as recommendations and exercise tips in improving patients' eating habits.

In [20] a patient monitoring system using IoT is proposed. The patient monitoring system is associated with a single concept called IoT. The Internet of Things is more than a connection between objects that have embedded technology with Internet infrastructure. Through using a wireless sensor network, smart monitoring and reliable connection between things can be achieved. Collecting data and information is a major challenge in wireless networking (sensors). The purpose of this article is to obtain efficient data from sources (patients) to the destination (physicians) through collecting data and disseminating information. The goal of data collection is to increase energy efficiency and disseminate information for data routing in the sensor network to reduce delays in data transmission from source to destination.

In [21] an intelligent patient monitoring system is proposed to monitor patients' health. Several sensors are used to collect a patient's biological behavior. Significant biological information is then transferred to the IoT cloud. The system proposed in this paper allows physicians and nurses to monitor patients in the Intensive Care Unit in real-time, which improves the efficiency and quality of services. There is a great opportunity to change this system as a wearable device that allows us to monitor the elderly or children from a remote distance.

In [22] new generation technology and the Internet of Things have been used to manage and analyze a large amount of data. The main focus of this research was on patients' ehealth and the IoT platform. By simulating their proposed method, they succeeded in achieving a significant improvement in patient health monitoring and analysis. 
In [23] examines the health care monitoring system and IoT-based analysis. In this research, an innovative IoT-based framework for health applications has been successfully developed. The system can accurately measure three vital signs, including body temperature, heart rate, and blood pressure. Data is available for physicians to observe and monitor in real-time, even if patients take tests out of the clinical environment.

In [24] a practical solution is proposed for the use, development of fog computing features, and smart e-health gateways in the field of IoT-based health systems. A medical case study titled Score Warning Early was used to monitor patients with acute illnesses.

In [25] a model for effective management of cardiac patient data is proposed. This model of resource management which is based on fog computing uses the iFog Sim tool to analyze the performance of the proposed model in the cloud environment.

In [26] provides information on fog computing, how they relate to other computational patterns such as Cloudlets, MEC, and edge computing. The classification of research topics has been done in May calculations.

In [27] a combination of X-Means algorithm, group learning system, and N-List structure is provided to analyze a mobile telecommunication company's customer portfolio. The X-Means algorithm is used to determine the optimal number of clusters and customer clusters in a mobile telecommunication company. The ensemble learning algorithm is used to assign categories to newer customers and the N-List structure is used to analyze the customer portfolio.

In [28] the researcher has been able to provide a method for analyzing the customer portfolio using clustering techniques, deep neural network, and online hybrid similarity 
criteria. However, each of the proposed methods has challenges such as inaccuracy and high error of recommendations.

Table 1. Comparing the advantages and disadvantages of previous methods

\begin{tabular}{|c|c|c|c|}
\hline reference & Method & Disadvantage & Advantage \\
\hline [4] & $\begin{array}{l}\text { A new platform for the smart } \\
\text { home to analyze IoT big data } \\
\text { with cloud computing } \\
\text { technology }\end{array}$ & Insufficient accuracy & Energy conservation \\
\hline [5] & $\begin{array}{l}\text { Provide a patient remote } \\
\text { monitoring system with the } \\
\text { aim of effectively managing } \\
\text { hospital resources through } \\
\text { patient monitoring at home }\end{array}$ & $\begin{array}{l}\text { Insufficient accuracy of } \\
\text { diagnostic information, } \\
\text { high classification error }\end{array}$ & $\begin{array}{l}\text { High classification speed, } \\
\text { removing outliers, using } \\
\text { IoT }\end{array}$ \\
\hline [10] & $\begin{array}{l}\text { An innovative IoT-based } \\
\text { system for identifying } \\
\text { medications and monitoring } \\
\text { prescription medication }\end{array}$ & $\begin{array}{l}\text { Insufficient accuracy of } \\
\text { diagnostic information } \\
\text { high classification error }\end{array}$ & $\begin{array}{l}\text { High computational speed, } \\
\text { simple model, removing } \\
\text { outliers }\end{array}$ \\
\hline [11] & $\begin{array}{l}\text { A remote monitoring and } \\
\text { decision support system to } \\
\text { assist physicians in } \\
\text { diagnosing, remote } \\
\text { monitoring, treating, } \\
\text { prescribing, rehabilitating, } \\
\text { and advancing patients with } \\
\text { Parkinson's disease. }\end{array}$ & $\begin{array}{l}\text { complicated model, long } \\
\text { computation time }\end{array}$ & $\begin{array}{l}\text { High classification } \\
\text { accuracy, simple model }\end{array}$ \\
\hline [12] & $\begin{array}{l}\text { An IoT-based health } \\
\text { monitoring system } \\
\text { considering the role of smart } \\
\text { data in the smart home for } \\
\text { patient-centered remote health } \\
\text { monitoring }\end{array}$ & $\begin{array}{l}\text { Insufficient accuracy of } \\
\text { diagnostic information, } \\
\text { high classification error }\end{array}$ & High classification accuracy \\
\hline [13] & $\begin{array}{l}\text { An IoT-based mobile gateway } \\
\text { (e.g. mobile/tablet /PDA, etc.) } \\
\text { for health scenarios }\end{array}$ & $\begin{array}{l}\text { complicated model, long } \\
\text { computation time }\end{array}$ & $\begin{array}{l}\text { High accuracy in } \\
\text { classification, simple model }\end{array}$ \\
\hline [14] & $\begin{array}{l}\text { IoT-based health monitoring } \\
\text { system for children with } \\
\text { Autism }\end{array}$ & $\begin{array}{l}\text { complicated model, long } \\
\text { computation time }\end{array}$ & $\begin{array}{l}\text { High accuracy in } \\
\text { classification, simple model }\end{array}$ \\
\hline [15] & $\begin{array}{l}\text { An industrial IoT-based } \\
\text { monitoring framework for } \\
\text { healthcare }\end{array}$ & $\begin{array}{l}\text { High error, complicated } \\
\text { model and low } \\
\text { computational speed }\end{array}$ & $\begin{array}{l}\text { Optimal accuracy in } \\
\text { classification }\end{array}$ \\
\hline [16] & $\begin{array}{l}\text { Smart Architecture for In- } \\
\text { Home Healthcare }\end{array}$ & $\begin{array}{l}\text { Insufficient accuracy of } \\
\text { diagnostic information, } \\
\text { high classification error }\end{array}$ & $\begin{array}{l}\text { High computational speed, } \\
\text { simple model, removing } \\
\text { outliers }\end{array}$ \\
\hline [17] & $\begin{array}{l}\text { An IoT-based patient } \\
\text { monitoring framework in the } \\
\text { intensive care unit }\end{array}$ & $\begin{array}{l}\text { complicated model, long } \\
\text { computation time }\end{array}$ & $\begin{array}{l}\text { High accuracy in } \\
\text { classification, simple model }\end{array}$ \\
\hline [18] & $\begin{array}{l}\text { medication reminder and } \\
\text { monitoring system for health } \\
\text { using IoT }\end{array}$ & $\begin{array}{l}\text { Insufficient accuracy of } \\
\text { diagnostic information, } \\
\text { high classification error }\end{array}$ & $\begin{array}{l}\text { High computational speed, } \\
\text { simple model, removing } \\
\text { outliers }\end{array}$ \\
\hline
\end{tabular}




\begin{tabular}{|c|c|c|c|}
\hline [19] & $\begin{array}{l}\text { IoT based patient monitoring } \\
\text { system }\end{array}$ & $\begin{array}{l}\text { complicated model, long } \\
\text { computation time }\end{array}$ & $\begin{array}{l}\text { High accuracy in } \\
\text { classification, simple model }\end{array}$ \\
\hline [20] & $\begin{array}{l}\text { Patient monitoring system } \\
\text { using IoT }\end{array}$ & $\begin{array}{l}\text { Insufficient accuracy of } \\
\text { diagnostic information, } \\
\text { high classification error }\end{array}$ & $\begin{array}{l}\text { High accuracy in } \\
\text { classification }\end{array}$ \\
\hline [21] & $\begin{array}{l}\text { A smart patient monitoring } \\
\text { system to monitor patients' } \\
\text { health }\end{array}$ & $\begin{array}{l}\text { High error, complicated } \\
\text { model and low } \\
\text { computational speed }\end{array}$ & $\begin{array}{l}\text { Optimal classification } \\
\text { speed, removing outliers } \\
\text { and using IoT platform }\end{array}$ \\
\hline [22] & $\begin{array}{l}\text { New generation technology } \\
\text { and IoT for managing and } \\
\text { analyzing big data }\end{array}$ & $\begin{array}{l}\text { Insufficient accuracy of } \\
\text { diagnostic information, } \\
\text { high classification error }\end{array}$ & $\begin{array}{l}\text { Optimal accuracy in } \\
\text { classification }\end{array}$ \\
\hline [23] & $\begin{array}{l}\text { An IoT-based healthcare } \\
\text { monitoring and analysis } \\
\text { system }\end{array}$ & $\begin{array}{l}\text { High error, complicated } \\
\text { model and low } \\
\text { computational speed }\end{array}$ & $\begin{array}{l}\text { Optimal accuracy in } \\
\text { classification }\end{array}$ \\
\hline [24] & $\begin{array}{l}\text { A practical solution for use, } \\
\text { Developing features of fog } \\
\text { computing and smart e-health } \\
\text { gateways for IoT-based health } \\
\text { systems }\end{array}$ & $\begin{array}{l}\text { Insufficient accuracy of } \\
\text { diagnostic information, } \\
\text { high classification error }\end{array}$ & $\begin{array}{l}\text { Optimal classification } \\
\text { accuracy }\end{array}$ \\
\hline [25] & $\begin{array}{l}\text { A model for effective } \\
\text { management of cardiac } \\
\text { patient data with the iFogSim } \\
\text { tool in the cloud computing } \\
\text { environment }\end{array}$ & $\begin{array}{l}\text { complicated model, long } \\
\text { computational speed }\end{array}$ & $\begin{array}{l}\text { High classification accuracy } \\
\text { Simple model }\end{array}$ \\
\hline [26] & $\begin{array}{l}\text { Things to know about fog } \\
\text { computing and the pattern of } \\
\text { edge computing }\end{array}$ & Examine edge computing & $\begin{array}{l}\text { Papers are "review article" } \\
\text { type }\end{array}$ \\
\hline [27] & $\begin{array}{l}\text { Deep neural network } \\
\text { algorithm to determine user } \\
\text { categories }\end{array}$ & $\begin{array}{l}\text { Insufficient accuracy in } \\
\text { diagnosis }\end{array}$ & High computational speed \\
\hline [28] & $\begin{array}{l}\text { Using clustering techniques, } \\
\text { deep neural networks, online } \\
\text { hybrid similarity criteria as a } \\
\text { method for analysis }\end{array}$ & long computation time & High classification accuracy \\
\hline
\end{tabular}

According to the literature and the research done in the field of remote monitoring of patients' condition, it was observed that despite the different advantages of each proposed method, they have not yet been able to meet challenges like insufficient accuracy and high classification error. In this article, the LSTM deep neural network is used to remotely classify and monitor the health status of patients. Data information is provided as input to the deep neural network. Deep neural network is trained based on input data and produces a model based on hidden layers and neural structure. Then, it receives information about the patients' condition and uses the generated model to remotely classify and monitor patients' health status. 


\section{The architecture of the proposed model}

In this section, the architecture of the proposed model is described. The following figure shows the architecture of the proposed method. As shown in Figure (1), patients are hospitalized at home or in the hospital wearing wrist sensors. These sensors collect the necessary information and send them to cloud computing servers. Sensors used in the proposed architecture include heart rate sensor, body temperature sensor, blood pressure sensor, blood sugar sensor, stress sensor, consciousness sensor, pulse counter, and accelerometer. These sensors are connected to the microcontroller using wires. The communication between the sensors and the microcontroller can also be wireless and via Wi-Fi. The number of sensors is not limited and can be increased.

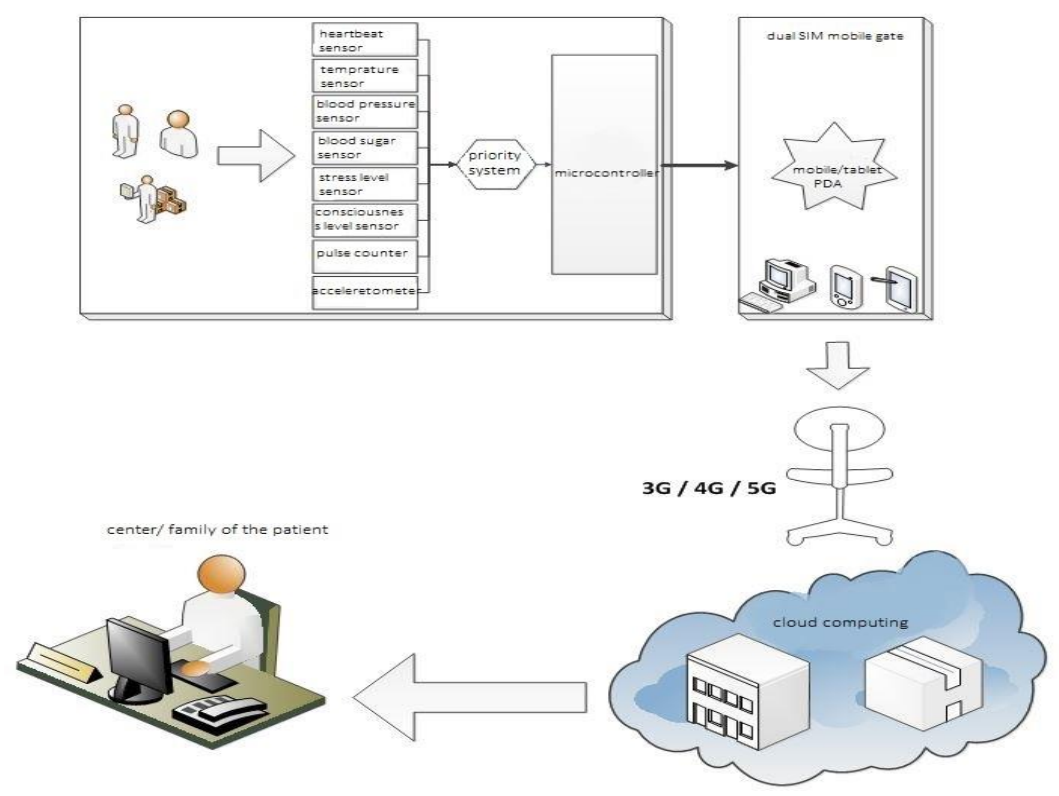

Fig 1. The architecture of the proposed model

In this paper, a system called "prioritization system" is considered as an IoT-based interface that detects more sensitive information and transmits it to the next level (cloud 
computing). The priority is attached to the data as a label and will be used in the next steps. Therefore, the information is sent to the IoT-based priority system and the priority system forms a queue based on the priority of sensitive and non-sensitive information and sends the queue to the microcontroller at specific time intervals. The microcontroller is used to monitor the parameters of the sensors and as an interface to send the information from the patient to the mobile gateway (e.g. mobile / tablet / PDA, etc.). The microcontroller then encrypts the information with AES and sends it to the mobile gateway via Bluetooth or Wi-Fi. The mobile gateway, which is equipped with two SIM cards, sends it to cloud computing through Next Generation Networks (5G).

The LSTM deep neural network system is embedded at the core of cloud computing to remotely classify and monitor patients' health status. The main task of the LSTM deep neural network algorithm is to determine the normal and acute conditions of patients. If the patient is in a severe condition based on the information received, the patient's deep neural network LSTM is detected and the relevant alarms are immediately sent to the patient, physician, nurse, and patient's family. Priority is given to patients with an acute condition.

\section{Description of the proposed method}

The most important parts of the proposed method in this article will be presented in the following sections.

\section{Sensors}

In [6], 3 sensors have been used to monitor patients' health status. Unlike the reference [6], instead of using only 3 sensors, we have used 8 sensors for patients. These sensors are as follows:

- Heart rate sensor 
- Body temperature sensor

- Blood pressure sensor

- Blood sugar level sensor

- Stress level sensor

- Consciousness level sensor

- Pulse counter

- Accelerometer

In the method presented in [6], only pulse counter, accelerometer, and temperature sensor are used, which cannot report the different states of the patient and this may lead to disorders in the treatment process. Therefore, in this article, we used more sensors to have an accurate analysis. The significant improvement that we have achieved in the new architecture is that, firstly, we have used more sensors and, secondly, we have used a priority system.

Sensors that are installed in different parts of the body to maintain patients' health are of great importance and based on the current condition of the person, the priority of that sensor should be defined for the priority system. For example, body temperature, stress level, and blood pressure of a person with breast cancer are prioritized over other signs. This is also very important for other patients, especially patients with rare diseases. Therefore, the priority system makes it possible that the sensors send the information to the mobile gateway (e.g. mobile / tablet / PDA, etc.) based on the degree of sensitivity. For example, the pulse counter and heart rate sensor report pulse count information to the microcontroller every minute. The body temperature sensor also provides information about the patient's body temperature every 30 seconds. Other sensors also provide specific behavior as needed. 
In the next step, the information is sent to the mobile gateway via Bluetooth and $\mathrm{Wi}$ Fi. By installing this feature, we can take steps in energy consumption and reliability of sending information, as well as preventing data loss. In the following, this issue will be fully discussed.

\section{Microcontroller interface (Single node)}

The microcontroller is used to monitor the parameters of the sensors and as an interface to send the information from the patient to the mobile gateway (e.g. mobile / tablet / PDA, etc.). The following section examines the process of encrypting and decrypting information using the AES algorithm.

\section{Mobile gateway}

In the next step, the encrypted information is sent from the microcontroller to the mobile gateway via Wi-Fi or Bluetooth. One of the features we have provided for the microcontroller is that sending data is possible both via Bluetooth and Wi-Fi together. In some cases, the mobile phone is located near the patient, and information is sent via Bluetooth to the mobile gateway. In cases where the mobile phone is located at a far distance, the information is sent via Wi-Fi.

\section{Reducing mobile energy consumption}

So far, we have discussed how to connect a microcontroller to a mobile gateway. Considering that reducing energy consumption is one of the most important criteria we consider in this process, we have improved the operation of sending information via mobile. The strategy we have considered in the mobile interface or gateway to improve power consumption is that the mobile device/tablet/PDA is not constantly connected to the microcontroller. Based on the time schedules, we turn on Wi-Fi when necessary. Also, we do not perform the process of encryption on the mobile device or tablet, etc. As fully 
described in the previous section, this process is performed by the microcontroller to reduce energy consumption as much as possible. On the other hand, calculations and any predictions are made by the mobile device and cloud computing data centers. Therefore, the amount of energy consumption will be reduced.

Also, since the mobile device is connected to the Internet and energy consumption is very high when the Internet is turned on, in this article, with the interface provided in the mobile device, when there is no need to send information to cloud computing, the Internet is turned off and the microcontroller is connected via Bluetooth. Except when Bluetooth has a limited range, communication is taken place via $\mathrm{Wi}-\mathrm{Fi}$, in which case the mobile phone Internet is turned off before sending information. Also, one of the other criteria we consider in this article is designing an architecture to produce a new and developed infrastructure with which we can provide a new model in the medical industry with the help of the Internet of Things.

\section{Providing two SIM cards for mobile}

One of the most important features that the article [8] does not consider is that the mobile device does not have two SIM cards. Having only one SIM card in critical situations can result in major damage or death. If we use only one SIM card and it faces problems in data connection, receiving information will be aborted. So, in this article, we use two SIM cards. Iranian telecommunications companies that offer SIM cards include Irancell, Hamrah Aval, and Rightel. Therefore, in critical situations, if the transmission of information faces problems, or one of the SIM cards does not work properly, the other SIM card starts working and the process of sending information continues. In such a situation, the reliability of the proposed model is approved. 


\section{5th generation mobile network or $5 G$}

In this article, we will apply the infrastructure for using the 5th generation mobile network so that the device which is equipped with this facility can use it. Moreover, we have used $3 \mathrm{rd}, 4^{\text {th }}$, and lower generations so that devices with lower capabilities can send messages. Therefore, in the proposed model, mobile gateways send their information to the cloud through the Internet and 5G by default. Cloud computing stores information and sends an alarm to the patient's family. Finally, the patient's family or the center takes necessary measures and guides the patient in critical situations.

\section{Classification of patients using LSTM deep neural network}

In this section, LSTM deep neural network algorithm is introduced for classification and monitoring of patients' health status. After applying patient's status data to the proposed system, the two important phases of the proposed method are as follows: the first part of the samples is used to train algorithm patterns in the deep learning system and the second part is used as test samples to test the proposed method to classify and monitor the condition of patients.

\section{Separating data and training samples}

One of the important phases that must be considered in the training of the LSTM deep neural network algorithm is the separation of the samples into two main parts. Data sampling is one of the stages of data mining that has been considered in the proposed approach. There are different sampling methods, three of the most important of which are:

- Random sampling

- Classified sampling

- Balanced sampling 
Random sampling is one of the simplest sampling methods. It acts randomly and separates samples from the original data to the desired extent. One of the disadvantages of this method is that it may not accurately reflect the whole dataset, and ultimately reduce the accuracy of data classification and validation of the proposed method. stratified sampling method is also one of the improved methods of random sampling method. This method does the sampling process based on probability and also selects the samples as a percentage. This method of sampling has its problems, too. For example, it may not select probability-based samples. In balanced sampling, one selects the required samples in a balanced way from the existing categories. This method does not have the problems of the previous methods and selects balanced samples or data from the available samples. The sampling method used in this paper is balanced method in order to separate training data and test data. Therefore, $70 \%$ of the samples are training samples and $30 \%$ are test samples.

\section{Applying LSTM deep neural network algorithm}

Deep learning neural network is one of the important categories in the field of classification and monitoring of patients' condition. The structure of this classification is examined in the following. 4 types of layers will be used in the neural network used in the proposed method, and we will discuss them below.

\section{Input layer}

The parameters semantically examined in the previous section are applied in a normalized way as the input of the deep neural network. The nerves of this layer represent the input data vector resulting from the feature extraction step.

\section{Hidden layers}


Generally, neural network training and the initial weights enter the network respectively in a supervised learning manner and Gaussian distribution (or normal distribution). By implementing backpropagation method, these weights are updated and reused in the network. This type of training is very slow and finding the local minimum instead of the general minimum has always been one of the challenges of this method. Hilton (2006) showed that we can solve this problem by using each layer pre-training employing Unsupervised Learning. Therefore, in this paper, deep neural network architecture is applied using the method proposed by Hilton. The deep neural network architecture as well as the related explanations used in this paper can be seen in Figure 2.

\section{Output layer}

In this section, two types of output layers are used. An output layer called output layer 1 is used in order to rebuild the network. This layer is made of input and is used only to train the neural network. This layer obtains its input information from the last layer of hidden layers and performs backpropagation. Another output layer that will be used in this article is soft max layer and will be referred to as output layer 2 . This layer is embedded in order to use regression. This method of data regression is a generalized logic regression.

This method is used when dealing with multiple data classes. The output of this method determines the probability of belonging to each class of data.

\section{Software regression function}

In logistic regression, the regression function has a nonlinear relationship with the linear combination of descriptive variables. This relationship is modeled with the Pro bit function. In definitions associated with classification, a binary variable is achieved [29].:

$y_{i} \in\{-1 .+1\}$ 
This is to regulate a Bernoulli Y random variable with $(\eta)$ probability of success. Success probability depends on a predictor, $\eta=\eta(\mathrm{x})$. If the predictor regulating a random variable is considered $\mathrm{X}$, then $\eta(\mathrm{x})$ is a conditional expectation of $\mathrm{Y}$. In such a case we have relation (2):

$E\{Y \mid X\}=\eta(x)$

In classification, however, a link function to the relation form (4) transfers the expectation to the linear composition of the predictors. Such models are called generalized linear models. When having logistic regression, the link function is selected logarithmically according to Equation (3).

$g(a)=\ln \frac{a}{1-a}$

The inverse of a logarithmic function is a logistic function that is selected as Equation (4):

$h_{\theta}(x)=\frac{1}{1+\exp \left(-\theta^{T} \cdot x\right)}$

In this method, the model parameters $(\theta)$, are optimized by the loss function as Equation (5).

$(\theta)=-\left[\sum_{i=1}^{m}\left\{\begin{array}{c}y^{(i)} \cdot \ln \left(h_{\theta}\left(x^{(i)}\right)\right)+ \\ \left(1-y^{(i)}\right) \cdot \ln \left(1-h_{\theta}\left(x^{(i)}\right)\right)\end{array}\right\}\right]$ 


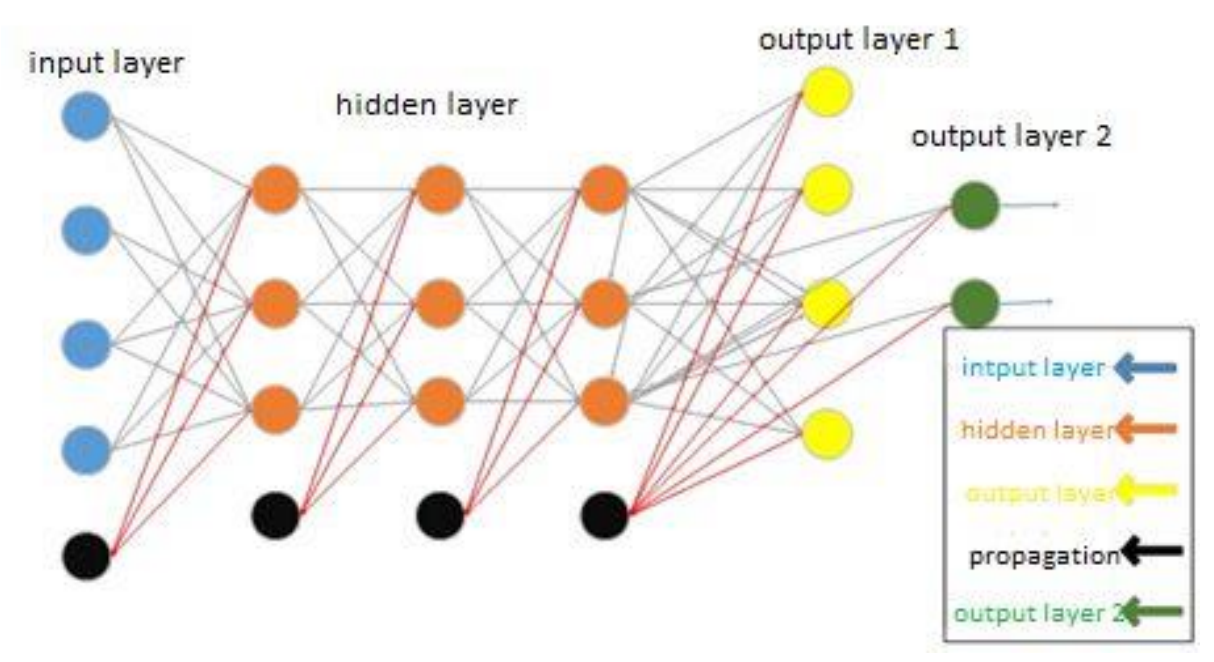

Fig 2. The basic architecture of deep neural network

Software regression is the generalized type of logistic regression and is used when there is more than one data class. According to the explanations given in this section, the neural network architecture used in this study can be shown according to Figure3. In this figure, the number of nerves in the input layer is assumed to be 4 , which might be different considering the characteristics obtained in the feature extraction stage. Each hidden layer includes 3 layers with 3 nerves, which may change during the execution phase depending on the response, accuracy, and speed of the network.

The performance of the proposed algorithm is based on deep learning. In this way, the training data set is entered into the core of the LSTM deep neural network algorithm and the model is generated. Test data is then entered into the model of the algorithm and makes a response for each test sample. Each response indicates whether the patient is in an acute condition or not. The response is either yes or no. The internal core of this algorithm is presented in the following format.

LSTM algorithms are performed by an inductive method which sorts complex polynomial models and selects the best solution using the so-called external criterion [29]. In Figure (2), the LSTM network structure used in the proposed model is provided. 

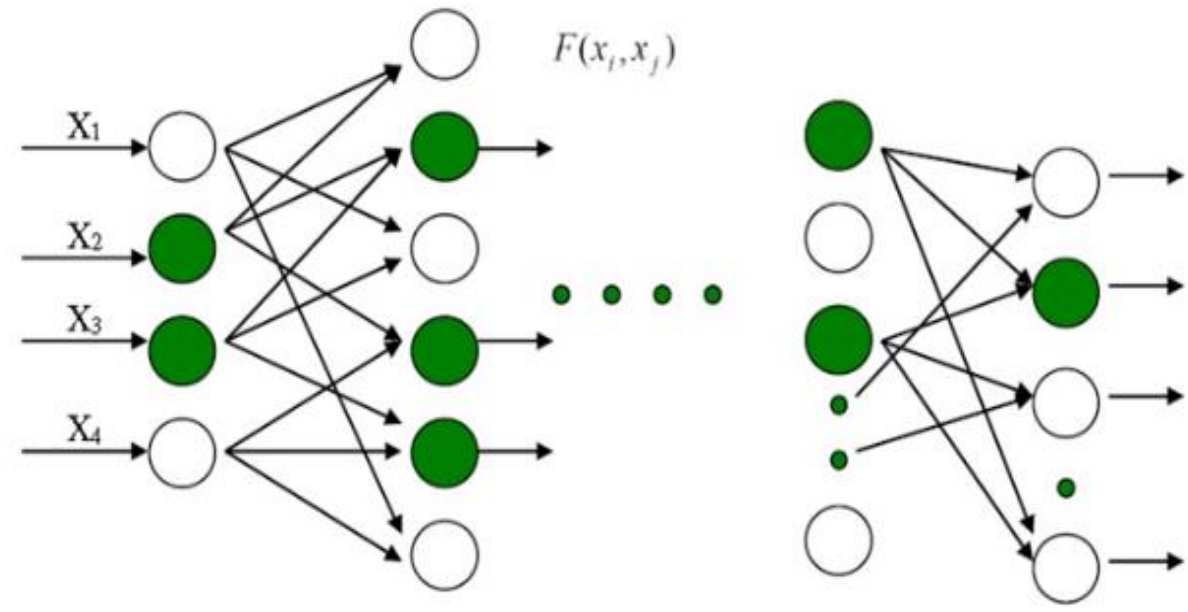

Correspond to selected node

Fig 3. The structure of LSTM used in the proposed model

As shown in Figure (3), $X=\left(x_{1}, x_{2}, \ldots, x_{n}\right)$ is entered into the artificial neural network as an input vector. Based on the $\mathrm{X}$ inputs, a set of nerves is formed in hidden layers and produces a neural network model. The artificial neural network includes an input vector, several hidden layers, and an output vector [30].

A set of neurons can represent the common LSTM algorithm. They have different pairs of nerve cells in each layer and join them using quadratic polynomials. The result of this connection is new neurons in the next layer. This method is defined to find a f defined function so that it can approach the actual output $\mathrm{y}$ for an input vector with data $X=$ $\left(x_{1} \cdot x_{2} \cdot x_{3} \ldots . x_{n}\right)$ Instead of the function $\mathrm{f}$ to predict output $\mathrm{y}$. Therefore, according to $\mathrm{M}$ samples from single-output multiple-input data pairs, the following relationships are defined:

$y_{i}=f\left(x_{i 1} \cdot x_{i 2} \cdot x_{i 3}, \ldots . x_{i n}\right) . i=1.2 \ldots . M$

An LSTM neural network might now be trained to predict output values. 
$X=\left(x_{i 1} \cdot x_{i 2} \cdot x_{i 3}, \ldots \cdot x_{i n}\right)$

Which means that:

$\hat{y}_{i}=\hat{f}\left(x_{i 1} \cdot x_{i 2} \cdot x_{i 3} \ldots . . x_{i n}\right) . i=1.2 \ldots . M$

Now in order to minimize the square of the difference between the predicted and the actual output, we face problem in determining a neural network of the deep neural network type:

$\sum_{k=1}^{M}\left[\left(\hat{f}\left(x_{i 1} \cdot x_{i 2} \cdot x_{i 3} \ldots . x_{i n}\right)-y_{i}\right)^{2}\right] \rightarrow$ Min.

The complex form polynomial used in the core of the deep neural network can be used to represent the general relationship between input and output variables. The complex polynomial is shown in relation (10):

$\hat{y}=a_{0}+\sum_{i=1}^{m} a_{i} x_{i}+\sum_{i=1}^{m} \sum_{j=1}^{m} a_{i j} x_{i} x_{j}+\sum_{i=1}^{m} \sum_{j=1}^{m} \sum_{k=1}^{m} a_{i j k} x_{i} x_{j} x_{k}+\cdots$

This is known as Ivakhnenko' s polynomial [30]. However, most programs use the twovariable form to predict the output y:

$\hat{y}=G\left(x_{i} \cdot x_{j}\right)=a_{0}+a_{1} x_{i}+a_{3} x_{i}^{2}+a_{4} x_{j}^{2}+a_{5} x_{i} x_{j}$

Using regression techniques, the coefficients $a_{i}$ in Equation (11) are calculated in order to calculate the difference between the actual returns $\hat{y} . \hat{y}$ is minimized for each pair $\left(x_{i}, x_{j}\right)$ as input. The variables actually show that a polynomial tree is constructed using the quadratic form in Equation (14), whose coefficients are obtained in least squares. Therefore, the coefficients of each quadratic function Gi are obtained to optimize the total input-output pair of the set:

$r^{2}=\frac{\sum_{i=1}^{M}\left(y_{i}-G_{i}\right)}{\sum_{i=1}^{M} y_{i}^{2}} \rightarrow M$ in 
$\left(y_{i} . i=1.2 \ldots . . M\right)$ The polynomial structure of regression in relation (12) can include the dependent samples $p . q \epsilon\{1.2 . \ldots . M\}$ for the difference $\left(\begin{array}{l}n \\ 2\end{array}\right)=n(n-1) / 2$ as the least squares $\left\{\left(y_{i} \cdot x_{i p} \cdot x_{i q}\right) .(i=1.2 \ldots . M)\right\}$ (This means that it is possible to construct a triple M data) $p$ ، $q \epsilon 1$ ، uses such examples.

$$
\left[\begin{array}{cccc}
x_{1 p} & x_{1 q} & \vdots & y_{1} \\
x_{2 p} & x_{2 q} & & y_{2} \\
\cdots & \cdots & \ldots & \cdots \\
x_{M p} & x_{M q} & \vdots & y_{M}
\end{array}\right]
$$

If a quadratic subunit is used in Equation (13), for each row of M data, the following matrix equation is obtained:

$\mathrm{Aa}=\mathrm{Y}$

Where a vector of unknown quadratic polynomial coefficients is in Equation (11):

$a=\left\{a_{0} \cdot a_{1} \cdot a_{2} \cdot a_{3} \cdot a_{4} \cdot a_{5}\right\}$

$Y=\left\{y_{1} \cdot y_{2} \cdot y_{3}, \ldots, y_{M}\right\}^{T}$

The vector is the output values of the samples:

$A=\left[\begin{array}{cccccc}1 & x_{1 p} & x_{1 q} & x_{1 p} x_{1 q} & x_{1 p}^{2} & x_{1 q}^{2} \\ 1 & x_{2 p} & x_{2 q} & x_{2 p} x_{2 q} & x_{2 p}^{2} & x_{2 q}^{2} \\ \vdots & \vdots & \vdots & \vdots & \vdots & \vdots \\ 1 & x_{M p} & x_{M p} & x_{M p} x_{M q} & x_{M p}^{2} & x_{M q}^{2}\end{array}\right]$ The least squares method of multiple regression analysis leads to the solving normal equations as follows:

$a=\left(A^{T} A\right)^{-1} A^{T} Y$

That the vector determines the best coefficients of the quadratic equation according to Equation (11) for the whole triple $\mathrm{M}$ data set. However, this solution, which is derived directly from normal equations, is almost capable of bypassing errors. Therefore, with 
this core, the process of model development, classifying, and monitoring of patients is done.

\section{Simulation results of the proposed method}

In this section, we first describe the system specifications for implementing the proposed method and then compare the results of the proposed method with those of other methods.

\section{System specifications for simulation}

The proposed method in this research has been implemented using MATLAB version 2015a. Table (2) also shows the specifications of the system in which the proposed method is implemented and the results are evaluated.

Table 2. system specifications for simulating and evaluating the results

\begin{tabular}{ll}
\hline Hardware/software & specifications \\
\hline Operating system & Windows 7 \\
\hline Type of operating system & 32-bit operating system \\
\hline RAM memory & 4Gb-3.06 usable \\
\hline processor & Intel processor-(Core $\left.{ }^{\mathrm{TM}}\right)$ i7 CPU7- Q720 @ $1.60 \mathrm{GHz} 1.60 \mathrm{GHz}$ \\
\hline
\end{tabular}

As can be seen in Table 2, the operating system used in this study is Windows 7 that is a 32-bit operating system. The RAM used is $4 \mathrm{~GB}-3.06 \mathrm{~GB}$ usable, the Intel processor - the number of cores is 7 (Core $\left.\left.{ }^{\mathrm{TM}}\right) \mathrm{i} 7 \mathrm{CPU}\right)$ - Q 720 @ $1.60 \mathrm{GHz}$ is $1.60 \mathrm{GHz}$.

\section{Dataset}

This study uses datasets associated with IoT devices that are connected to patients in the form of wrist sensors. Data received from IoT devices is distributed on an hourly basis. Therefore, about 10,000 data are received per hour and samples are more than 1 
terabyte. IoT servers connected to patients store data received from IoT devices in 24 hours. Table (3) shows an example of the dataset suggested in the proposed method.

Table 3. An example of the dataset suggested in the proposed method

\begin{tabular}{cccccc}
\hline $\begin{array}{c}\text { Patient } \\
\text { no. }\end{array}$ & Type of activity & Heartbeat rate & temperature & $\begin{array}{c}\text { Time of the } \\
\text { day }\end{array}$ & $\begin{array}{c}\text { Patients' } \\
\text { health } \\
\text { status }\end{array}$ \\
\hline 1 & sleep & 70 & 37 & 7 & normal \\
\hline 2 & lunch & 139 & 38 & 14 & critical \\
\hline 3 & Doing sport & 70 & 33 & 10 & normal \\
\hline 4 & sleep & 39 & 39 & 1 & critical \\
\hline 5 & Doing sport & 120 & 34 & 17 & normal \\
\hline 6 & Heavy activity & 124 & 37 & 14 & normal \\
\hline 7 & walking & 80 & 30 & 17 & critical \\
\hline 8 & sleep & 55 & 28 & 0 & critical \\
\hline 9 & awake & 110 & 38 & 10 & critical \\
\hline 10 & Moderate & 75 & 36 & 12 & normal \\
\hline & activity & & & & \\
\hline
\end{tabular}

As can be seen in Table 3, 10 individuals are examined and their health status is assessed considering different conditions in different times of the day. We can observe that tachycardia is not a significant criterion to determine critical condition during heavy activities or doing sports.

\section{Assessment criteria}

The following equations show the calculation of accuracy, precision and recall [28].

Accuracy $_{j}=\frac{\sum_{i=1}^{n} T P(i)+\sum_{i=1}^{n} T N(i)}{\sum_{i=1}^{n} T P(i)+F N(i)+F P(i)+T N(i)}$

Sensitivity $_{j}=\frac{\sum_{i=1}^{n} T P(i)}{\sum_{i=1}^{n} T P(i)+F N(i)}$

Specificity $_{j}=\frac{\sum_{i=1}^{n} T N(i)}{\sum_{i=1}^{n} F P(i)+T N(i)}$

TP (True Positive) indicates patients who are in a normal health condition and identified as normal by the proposed method. TN (True Negative) indicates patients who are in a normal health condition but identified as critical by the proposed method. FP (False 
Positive) indicated patients who are in critical health condition and identified as normal by the proposed method. Finally, FN (False Negative) indicates patients who are in a critical health condition and identified as critical by the proposed method. $\mathrm{n}$ indicates the number of samples and $\mathrm{i}$ is between 1 and $\mathrm{n}$ [27].

\section{Comparing the results}

In this section, the results obtained from the simulation carried out in accordance with the proposed model are discussed. In this paper, the obtained results are compared with the findings of [8] and [25]. In this section, the accuracy of the proposed algorithm in different states of the patient's body is discussed. We consider this criterion in our simulation and compare the obtained results with the results of this article. The following figure shows the final accuracy of the proposed model where the number of sensors is 8 and each sensor sends 100 events per unit of time to the microcontroller.

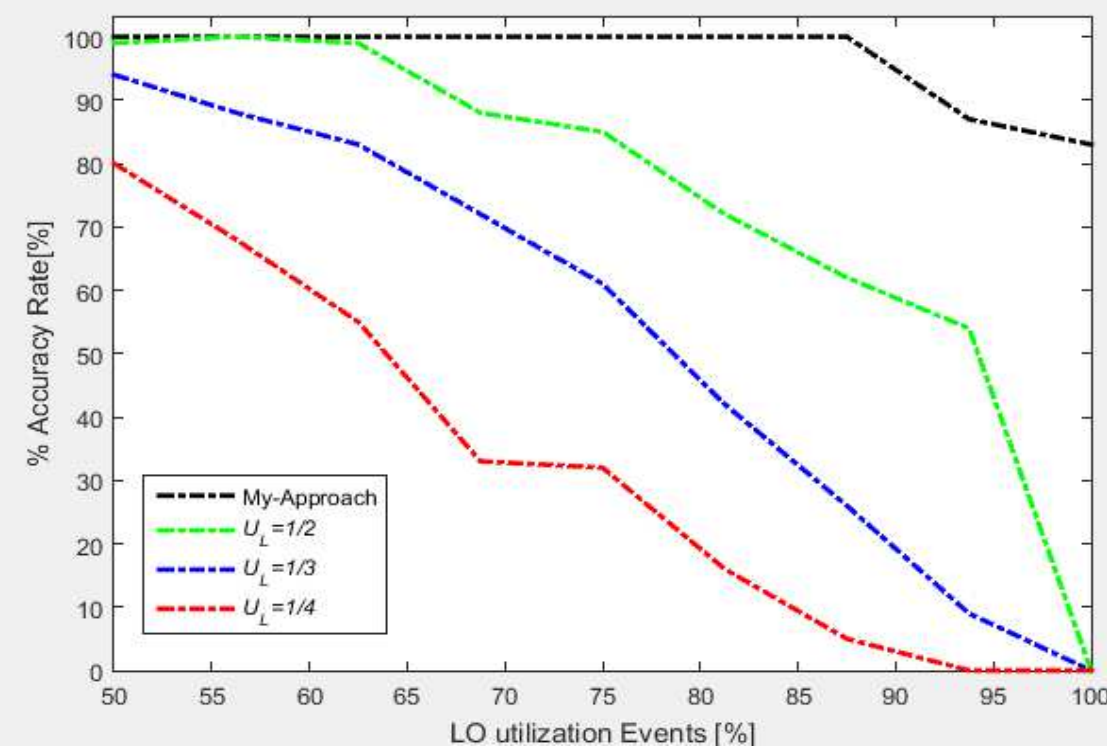

Fig 4: Comparison of patient health control accuracy of the proposed method using 8 sensors with that of other methods 
As can be seen in fig 4, the vertical axis indicates the degree of accuracy of patients' health control and the horizontal axis shows the percentage of response to events sent to the hospital center by sensors. The proposed model has $100 \%$ accuracy to respond and send information in $95 \%$ of different cases. While other methods with fewer sensors, such as 2, 3, and 4 sensors, have an accuracy of $90 \%$ to $10 \%$. One of the most important reasons for the high accuracy of the proposed method for sending and receiving information from sensors to the hospital center or to cloud computing is the use of the IoT technology and 5G platform. Also, the use of dual SIM mobile phones, wireless and wired communication channels has led to such accuracy. The following figure shows the test results of using 20 sensors compared to other methods.

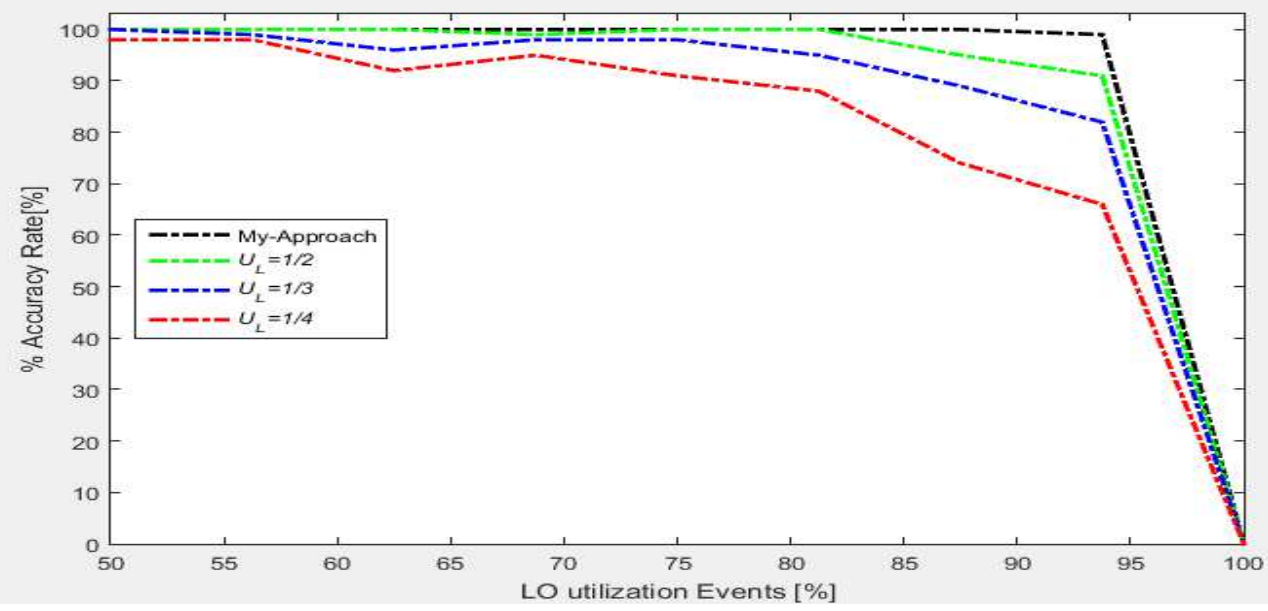

Fig 5: Results of the accuracy of sending and receiving data from sensors to the hospital in the proposed method using 20 sensors with that of other methods

As can be seen from Fig 5, the accuracy of sending and receiving data from sensors to the hospital center is $100 \%$ in $95 \%$ of cases and there is no challenges or problems regarding the data. 
Finally, we reduced the number of sensors on the patient's body so that we could measure the accuracy of diagnosing the patient's conditions. The following figure shows the accuracy level of diagnosing the condition of patients in the proposed method.

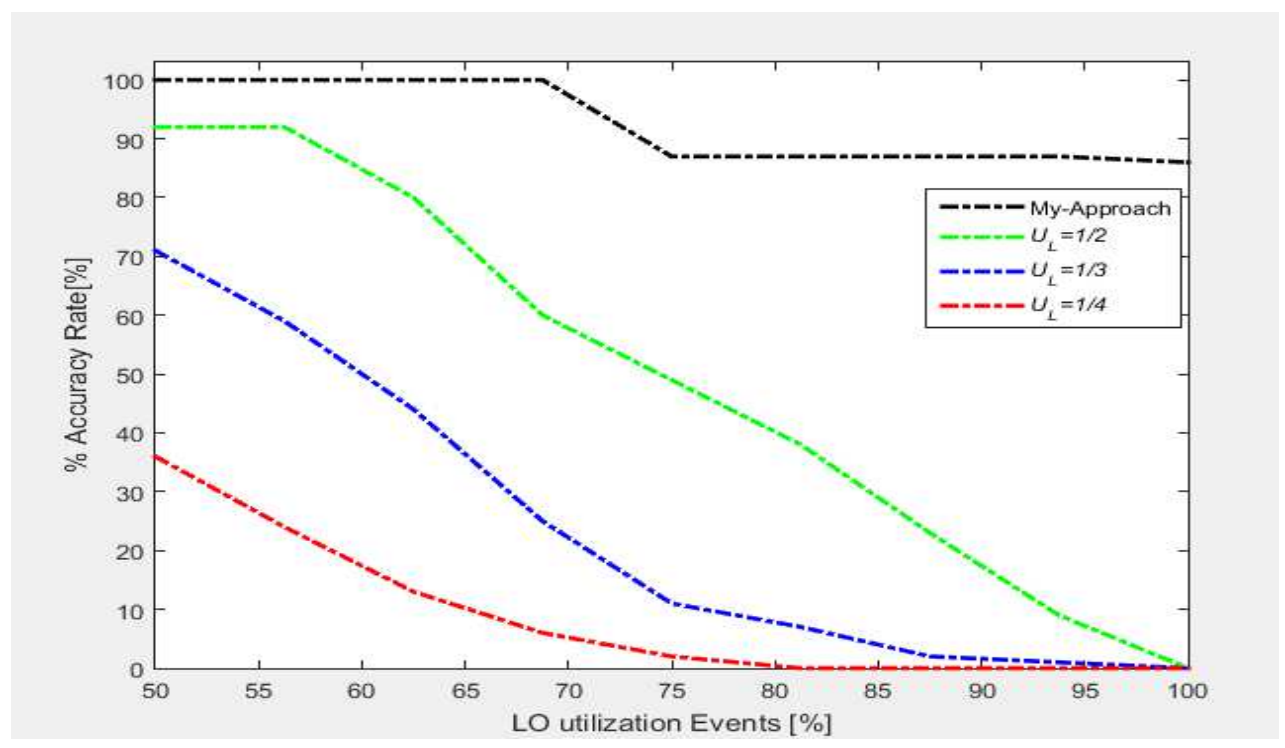

Fig6. The accuracy of classifying patients' health status in the proposed model

As we can see in Fig 6, the accuracy of the proposed method in diagnosing patients' condition in critical conditions is $100 \%$ in $70 \%$ of cases and $87 \%$ in $30 \%$ of cases. However, it can be concluded that the number of patient sensors should be increased as much as possible to increase the accuracy of diagnosis and control of the patients' condition.

Finally, we compare the final findings of this article with the results of the base article. The following figure shows the comparison of the accuracy of the proposed method with that of the reference [13]. 


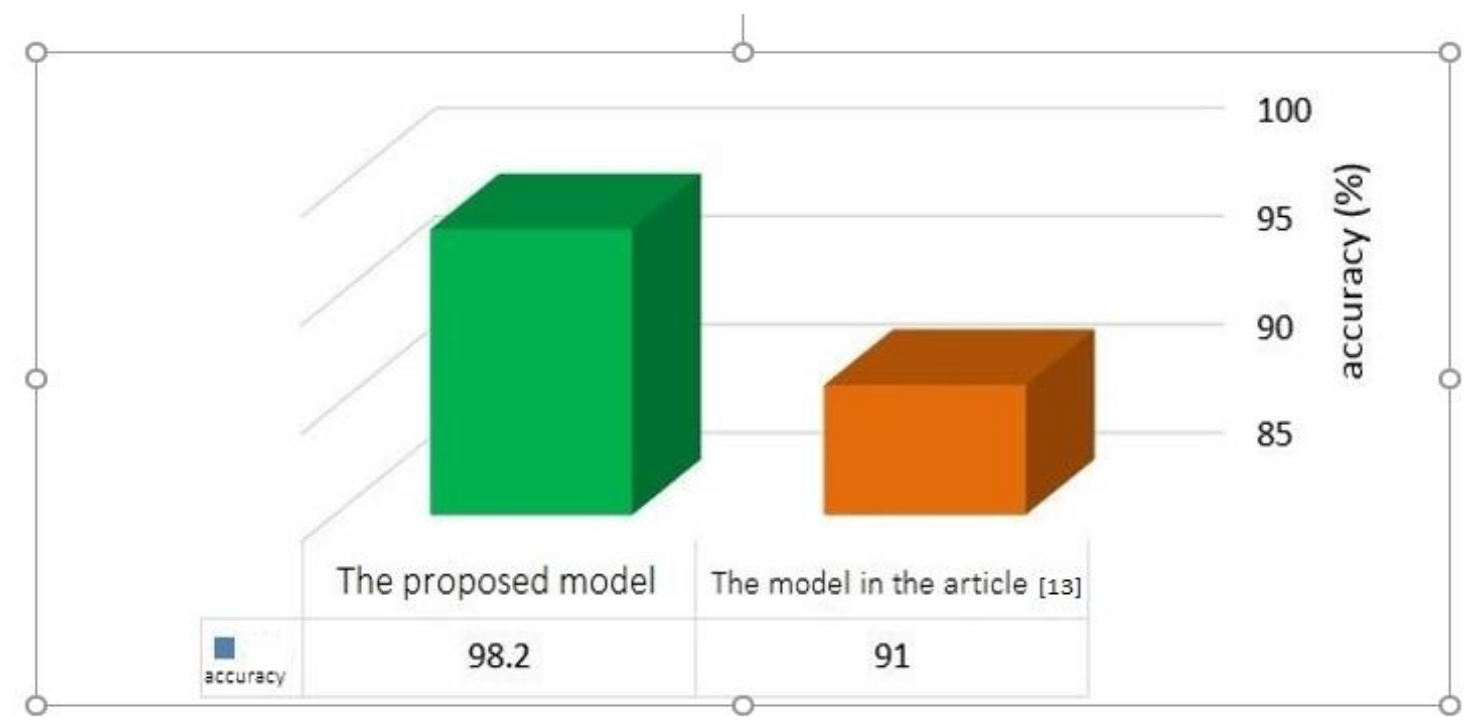

Fig 7. Comparison of classification accuracy and diagnosis accuracy of the proposed model with those of reference [13]

As can be seen in Fig 7, the accuracy of the proposed method with 8 sensors is equal to $98.2 \%$ and the accuracy of the method proposed in reference [13] is equal to $91 \%$. With these interpretations, the improvement rate of classification accuracy and diagnosis of patients in the proposed method compared to the method presented in reference [13] is about $7.2 \%$.

In this section, the results of the proposed method which are based on LTSM deep neural network are compared with that of [25] in terms of accuracy, precision, and recall. Table 4 shows the comparison of the results of the proposed method with other methods used in [25] in terms of accuracy, precision, and recall. 
Table 4. the comparison of the proposed method based on LTSM deep neural network with that of [25] in terms of accuracy, precision, and recall.

\begin{tabular}{ccccc}
\hline methods & Accuracy \% & Precision\% & Recall \% & Error \% \\
\hline SVM & 77.5 & & & \\
\hline Decision tree & 70.94 & 62 & 86 & 22.5 \\
\hline KNN & 76 & 56 & 83 & 28.06 \\
\hline Naïve Bayes Model & 76.60 & 55 & 83 & 24 \\
\hline Methods [25] & 86.72 & 62.2 & 89 & 13.38 \\
\hline Suggested method & 97.13 & 67 & 98.21 & 10.98 \\
\hline
\end{tabular}

As can be seen in Table 4, the accuracy level of the proposed model in identifying patients with critical health status is $97.13 \%$. The proposed method compared to SVM, decision tree, $\mathrm{KNN}$ and Naïve Bayes methods is $21.87 \%$ and the improvement rate of the proposed method compared to the method suggested in [25] is $10.41 \%$. The accuracy of identifying patients with critical health status in the proposed method is equal to $98.44 \%$. The proposed method is $39.64 \%$ compared to the SVM, Decision Tree, KNN, and Naïve Bayes methods and the improvement rate of the proposed method compared to the method suggested in [25] is $31.44 \%$. The recall of identifying patients with critical health status in the proposed method is $98.21 \%$. The proposed method is $14.96 \%$ compared to SVM, decision tree, KNN, and Naïve Bayes methods and the improvement rate of the proposed method compared to the method suggested in [25] is $9.21 \%$.

\section{Discussion and Future suggestions}

Patients who suffer from underlying and chronic diseases need special care which can be provided by a nurse, physician, hospital, and family. Home caregiving costs a lot and patients cannot stay in the hospital for a long time. In context-based systems, it is important to study sensitive tasks as well as provide a framework that can provide the process of analyzing, and implementing new methods in the medical industry. Therefore, 
in this paper, a combination of cloud computing systems and the Internet of Things has been used. At the core of the Internet of Things, the prioritization framework is used to prioritize sensitive requests, and in cloud computing, the LSTM deep neural network algorithm is used to remotely classify and monitor patients. By simulating and evaluating the results of the proposed method using MATLAB software, it was observed that the accuracy, precision, and recall of the proposed method compared to those of other methods have significantly improved.

To further develop this study and to improve the results of the proposed method, the use of semantic methods and optimization techniques such as Krill Herd Optimization algorithm, compact cat swarm optimization algorithm, or Harris hawk s optimization can be used in order to select the optimal features. Also, using reinforcement and collective learning systems instead of LSTM deep neural network algorithm can improve the results of this paper.

\section{Ethics approval and consent to participate}

Not applicable

\section{Consent for publication}

Not applicable

\section{Availability of data and materials}

The datasets generated and/or analysed during the current study are not publicly available due [REASON WHY DATA ARE NOT PUBLIC] but are available from the corresponding author on reasonable request.

\section{Competing interests}

The authors declare that they have no competing interests

\section{Funding}

Not applicable 


\section{Authors' contributions}

All authors contributed to developing the ideas, and writing and reviewing this manuscript. All authors read and approved the final manuscript.

\section{Acknowledgement}

Not applicable

\section{References}

1. Nan, Guofang, et al. "Distributed resource allocation in cloud-based wireless multimedia social networks." IEEE Network 28.4 (2014): 74-80.2. R. Yu, Y. Zhang, S. Gjessing, W. Xia, and K. Yang (2013) Toward cloud based vehicular networks with efficient resource management. IEEE Netw. Mag., 27(5), 48-55

3. Y. Gan and C. Delimitrou, "The Architectural Implications of Cloud Microservices," IEEE Computer Architecture Letters, vol. 17, no. 2, pp. 155-158, 2018.

4. Yassine, Abdulsalam, et al. "IoT big data analytics for smart homes with fog and cloud computing." Future Generation Computer Systems 91 (2019): 563-573.

5. S Suryandari, Yulia. "Survei IoT Healthcare Device." Jurnal Sistem Cerdas 3.2 (2020): 153164.

6. Mutlag, Ammar Awad, et al. "Enabling technologies for fog computing in healthcare IoT systems." Future Generation Computer Systems 90 (2019): 62-78.

7. Chrystinne Oliveira Fernandes (\&) and Carlos José Pereira de Lucena, “An Internet of Things Application with an Accessible Interface for Remote Monitoring Patients. ”, Springer International Publishing Switzerland 2015.

8. Geetha, G., and K. Mohana Prasad. "Survey on Real-Time Diabetic Patient's Monitoring Using Internet of Things." Artificial Intelligence Techniques for Advanced Computing Applications. Springer, Singapore 259-270.9. Qureshi, K. N., Din, S., Jeon, G., \& Piccialli, F. (2020). An accurate and dynamic predictive model for a smart M-Health system using machine learning. Information Sciences, 538, 486-502. 
10. Jara, Antonio J., Miguel A. Zamora, and Antonio F. Skarmeta. "Drug identification and interaction checker based on IoT to minimize adverse drug reactions and improve drug compliance." Personal and ubiquitous computing 18.1 (2014): 5-17.

11. Chiuchisan, I. U. L. I. A. N. A., and O. A. N. A. Geman. "An approach of a decision support and home monitoring system for patients with neurological disorders using internet of things concepts." WSEAS Transactions on Systems 13.1 (2014): 460-469.

12. Puustjärvi, Juha, and Leena Puustjärvi. "The role of smart data in smart home: health monitoring case." Procedia Computer Science 69 (2015): 143-151.

13. Santos, João, et al. "An IoT-based mobile gateway for intelligent personal assistants on mobile health environments." Journal of Network and Computer Applications 71 (2016): 194-204.

14. Kumar, KB Sundhara, and Krishna Bairavi. "IoT based health monitoring system for autistic patients." Proceedings of the 3rd International Symposium on Big Data and Cloud Computing Challenges (ISBCC-16'). Springer, Cham, 2016.

15. Hossain, M. Shamim, and Ghulam Muhammad. "Cloud-assisted industrial internet of things (iiot)-enabled framework for health monitoring." Computer Networks 101 (2016): 192-202.

16. Mano, Leandro Y., et al. "Exploiting IoT technologies for enhancing Health Smart Homes through patient identification and emotion recognition." Computer Communications 89 (2016): 178-190.

17. Bhatia, Munish, and Sandeep K. Sood. "Temporal informative analysis in smart-ICU monitoring: M-HealthCare perspective." Journal of medical systems 40.8 (2016): 1-15.

18. Zanjal, Samir V., and Girish R. Talmale. "Medicine reminder and monitoring system for secure health using IOT." Procedia Computer Science 78 (2016): 471-476.

19. Gupta, Sonam, Lipika Goel, and Abhay Kumar Agarwal. "A Novel Framework of Health Monitoring Systems." International Journal of Big Data and Analytics in Healthcare (IJBDAH) $6.1(2021): 1-14$.

20. Shaikh, Sohail, et al. "Patient monitoring system using iot." 2017 International Conference on Big Data, IoT and Data Science (BID). IEEE, 2017. 
21. Uddin, Mohammad Salah, Jannat Binta Alam, and Suraiya Banu. "Real time patient monitoring system based on Internet of Things." 2017 4th International Conference on Advances in Electrical Engineering (ICAEE). IEEE, 2017.

22. Dey, Nilanjan, Amira S. Ashour, and Chintan Bhatt. "Internet of things driven connected healthcare." Internet of things and big data technologies for next generation healthcare. Springer, Cham, 2017. 3-12.

23. Tan, E. T., and Z. Abdul Halim. "Health care monitoring system and analytics based on internet of things framework." IETE Journal of Research 65.5 (2019): 653-660.

24. Rahmani, Amir M., et al. "Exploiting smart e-Health gateways at the edge of healthcare Internet-of-Things: A fog computing approach." Future Generation Computer Systems 78 (2018): $641-658$.

25. Gill, Sukhpal Singh, et al. "Fog-based smart healthcare as a big data and cloud service for heart patients using IoT." International Conference on Intelligent Data Communication Technologies and Internet of Things. Springer, Cham, 2018.

26. A. Yousefpour, G. Ishigaki, and J. P. Jue, "Fog Computing: Towards Minimizing Delay in the Internet of Things,” in IEEE International Conference on Edge Computing (EDGE), 2017 27.Vahidi Farashah, M., Etebarian, A., Azmi, R. et al. An analytics model for TelecoVAS customers' basket clustering using ensemble learning approach. J Big Data 8, 36 (2021).

28. Vahidi Farashah, M., Etebarian, A., Azmi, R. et al. A hybrid recommender system based-on link prediction for movie baskets analysis. J Big Data 8, 32 (2021).

29Farlow, Stanley J. Self-organizing methods in modeling: GMDH type algorithms. CrC Press, 2020.

30. F Feng, Xiao, et al. "Artificial neural networks forecasting of PM2. 5 pollution using air mass trajectory based geographic model and wavelet transformation." Atmospheric Environment 107 (2015): 118-128. 
Figures
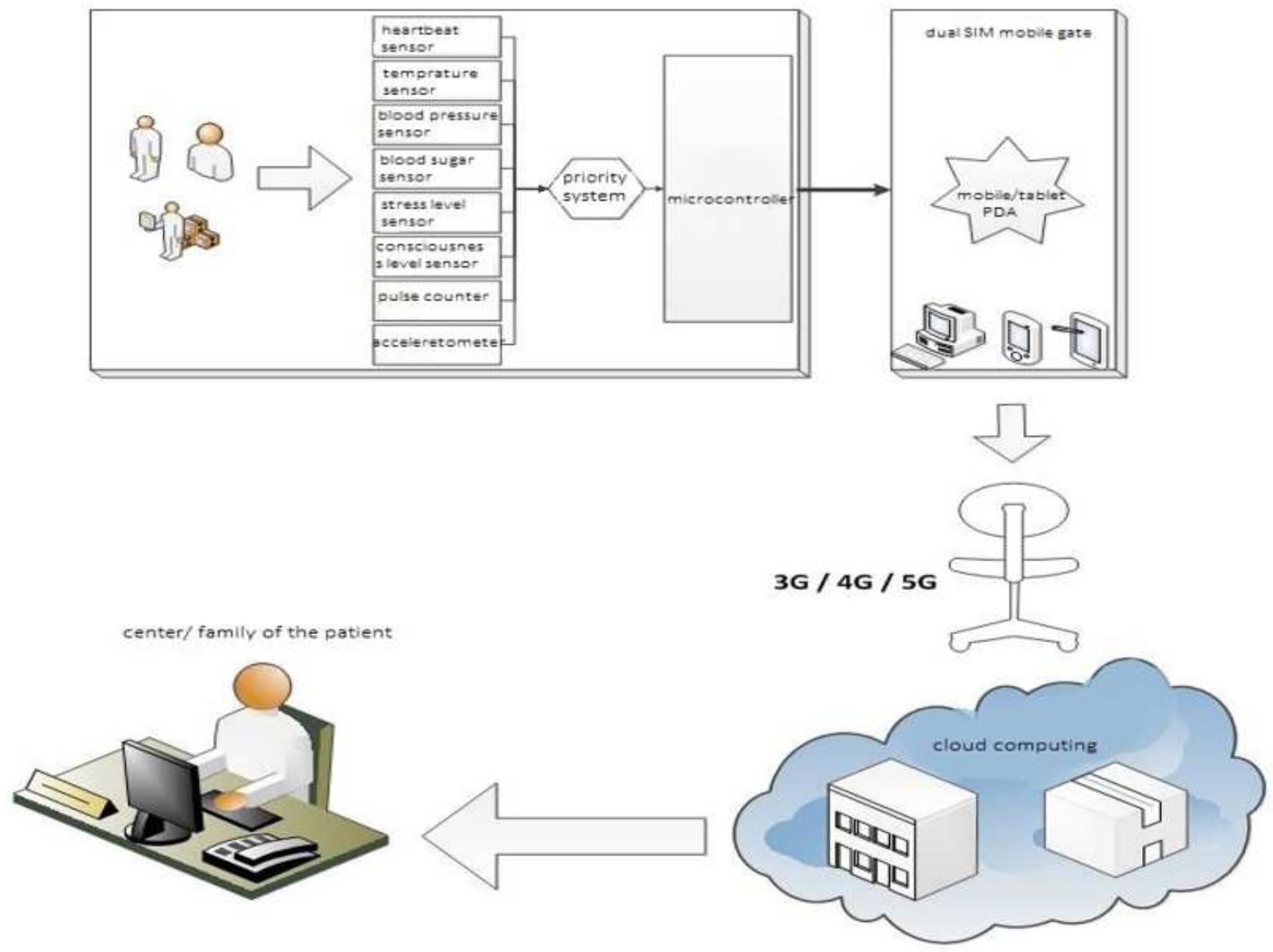

Figure 1

The architecture of the proposed model 


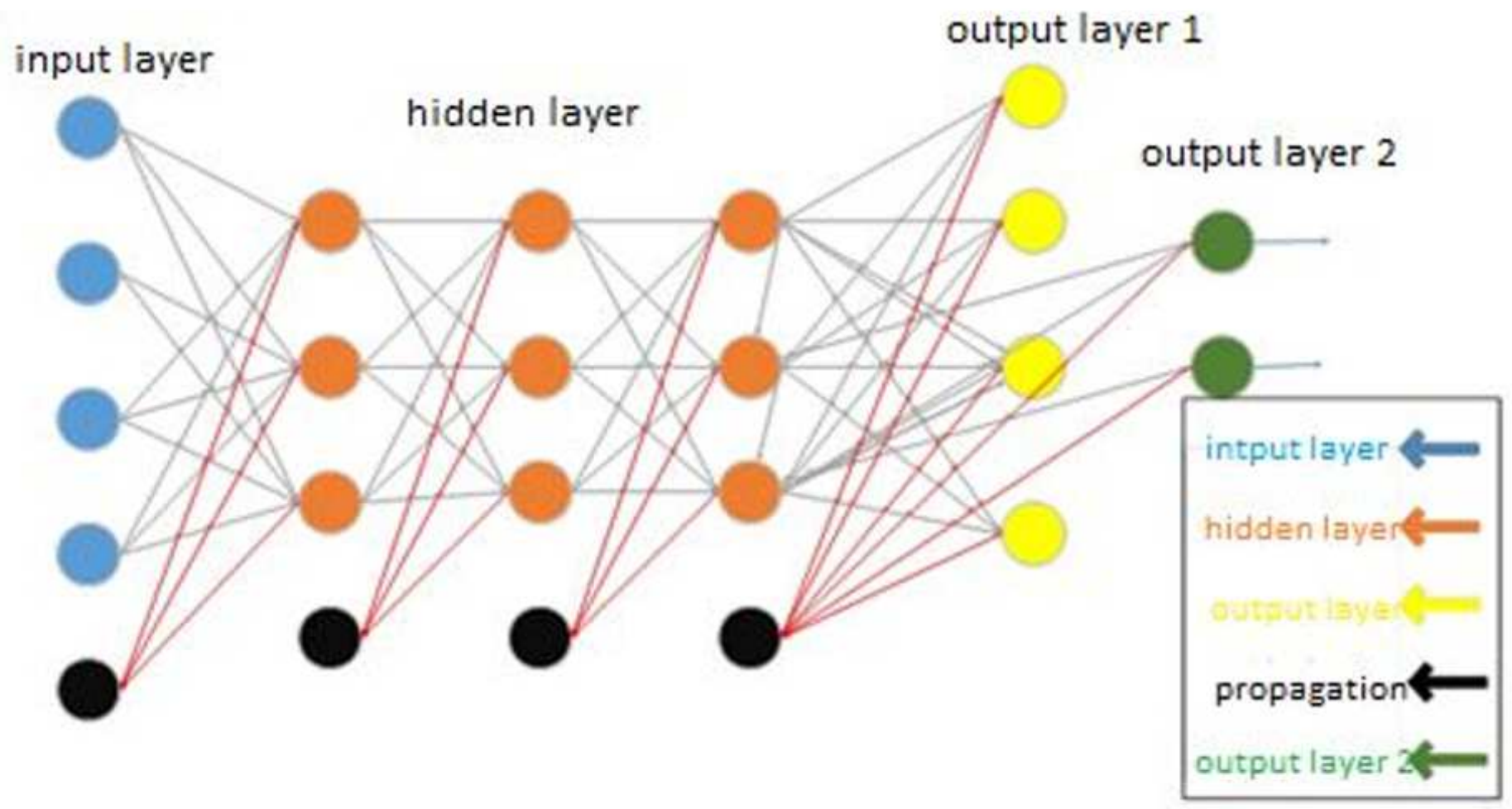

Figure 2

The basic architecture of deep neural network
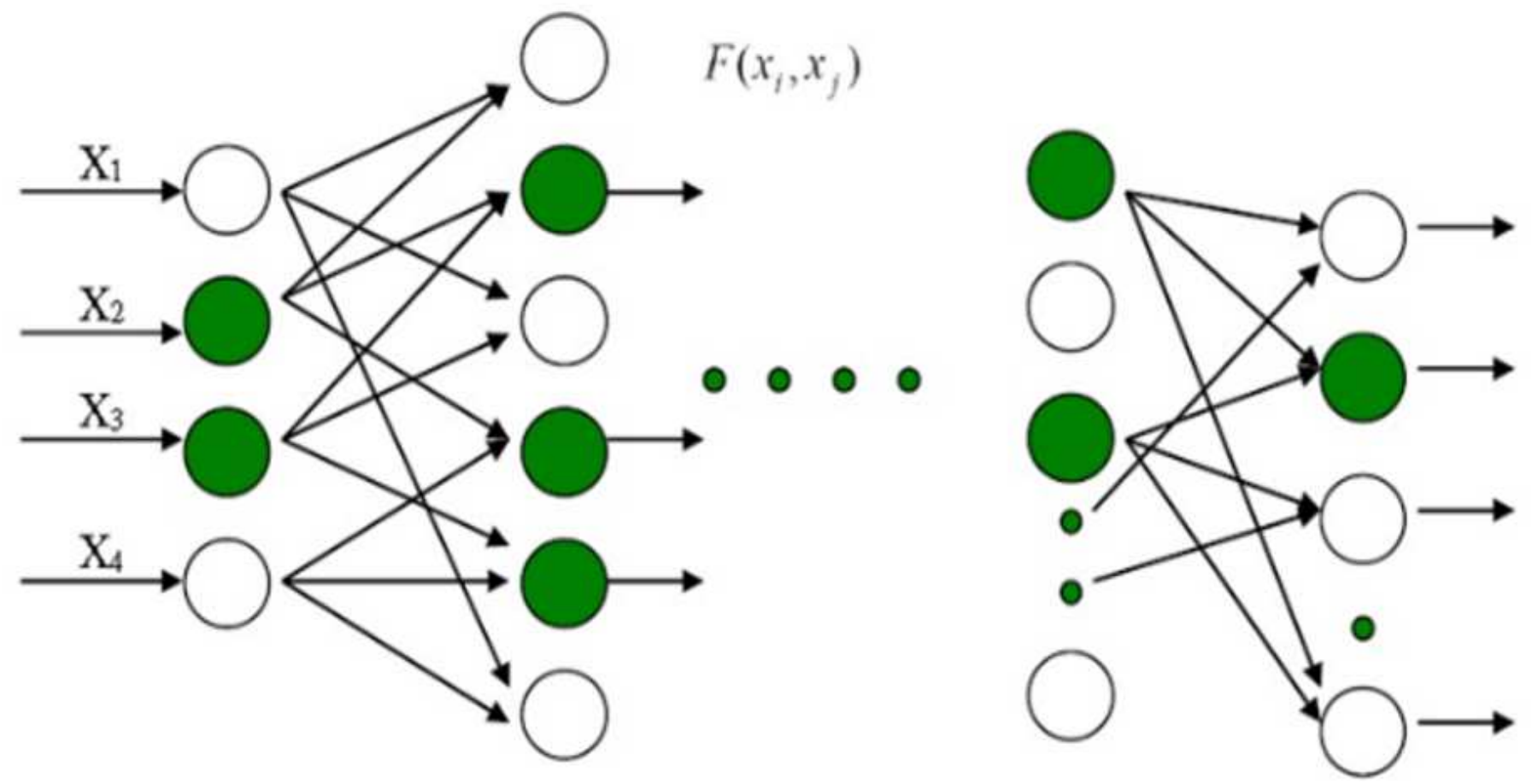

Correspond to selected node 
Figure 3

The structure of LSTM used in the proposed model

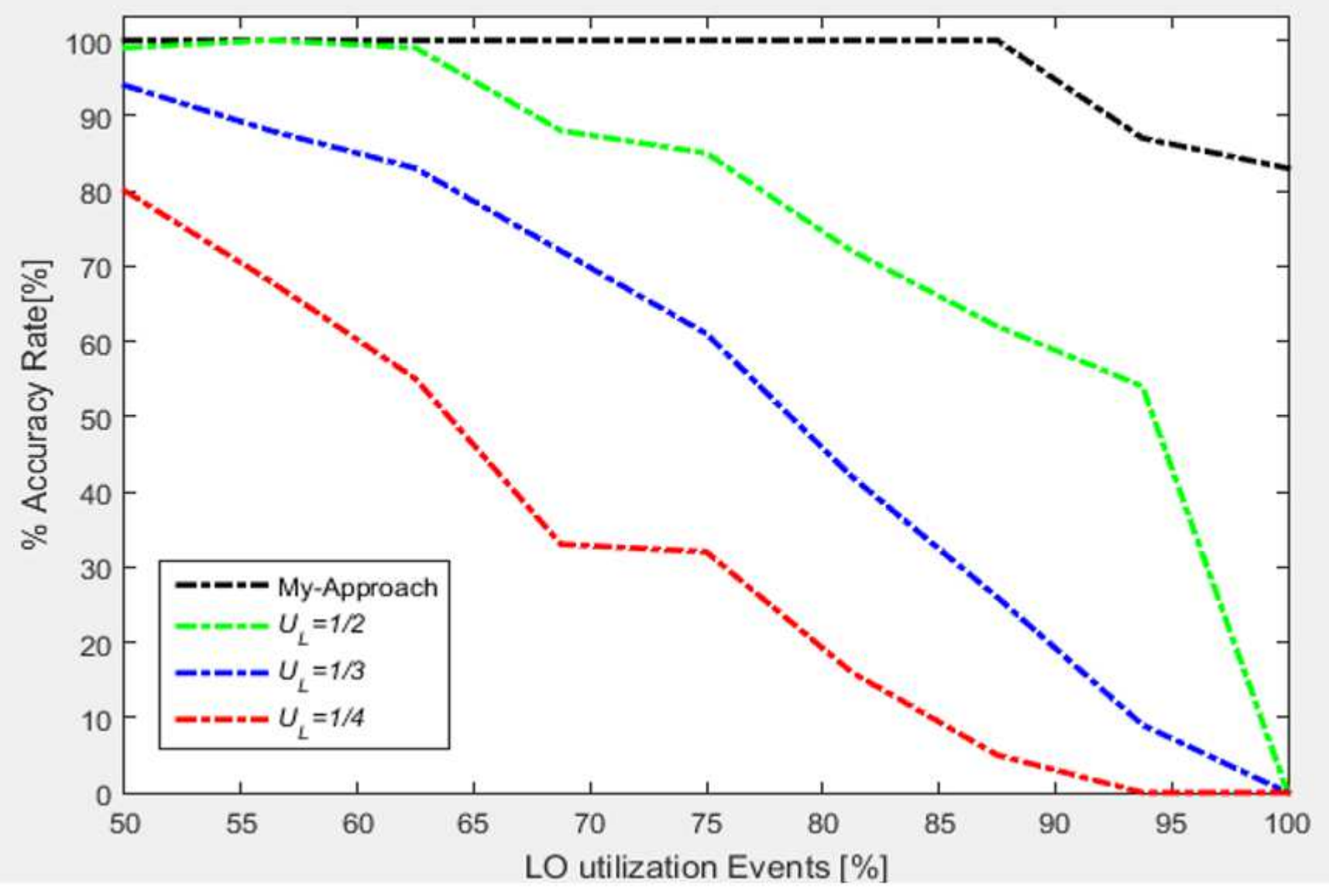

Figure 4

Comparison of patient health control accuracy of the proposed method using 8 sensors with that of other methods 


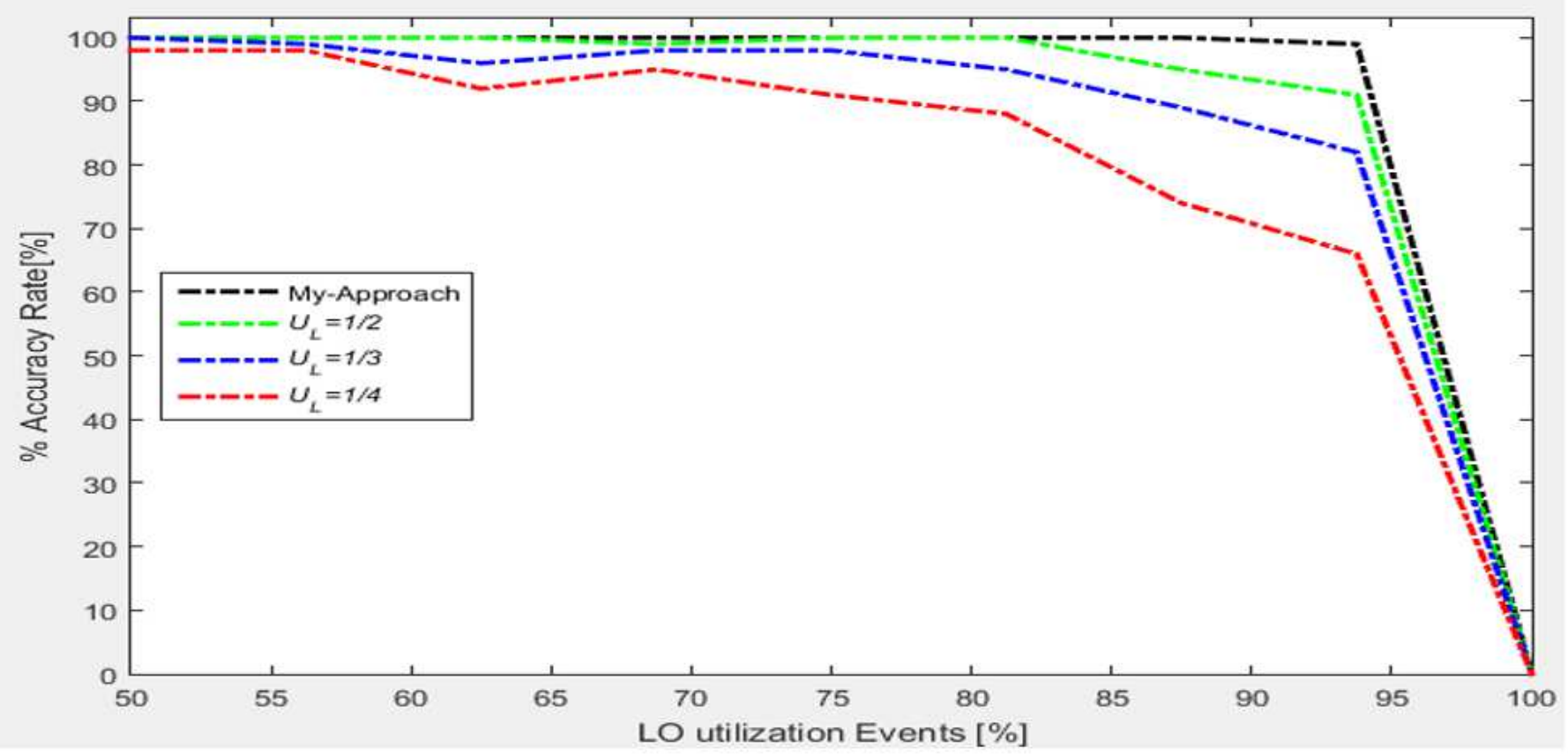

Figure 5

Results of the accuracy of sending and receiving data from sensors to the hospital in the proposed method using 20 sensors with that of other methods

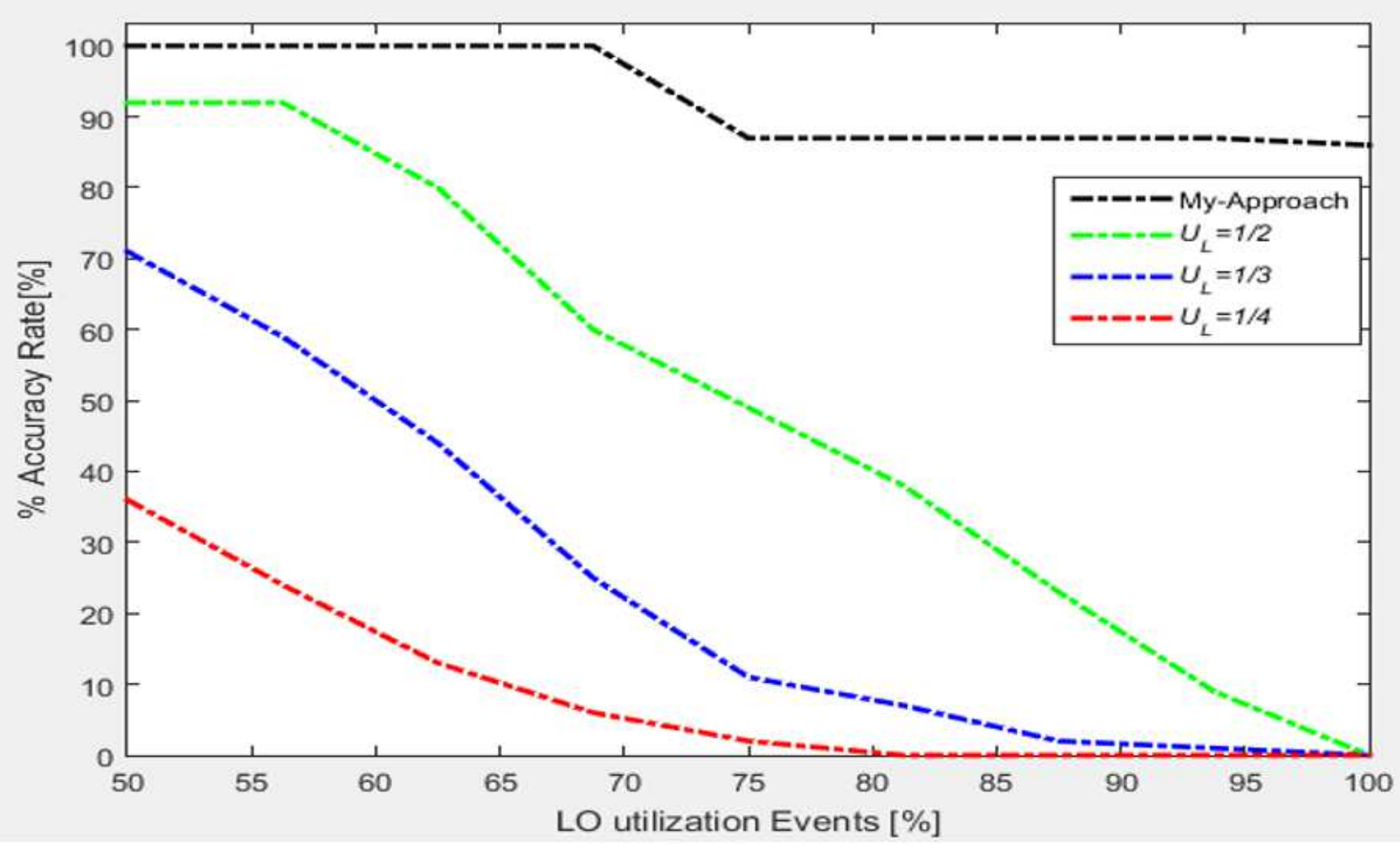

Figure 6 
The accuracy of classifying patients' health status in the proposed model

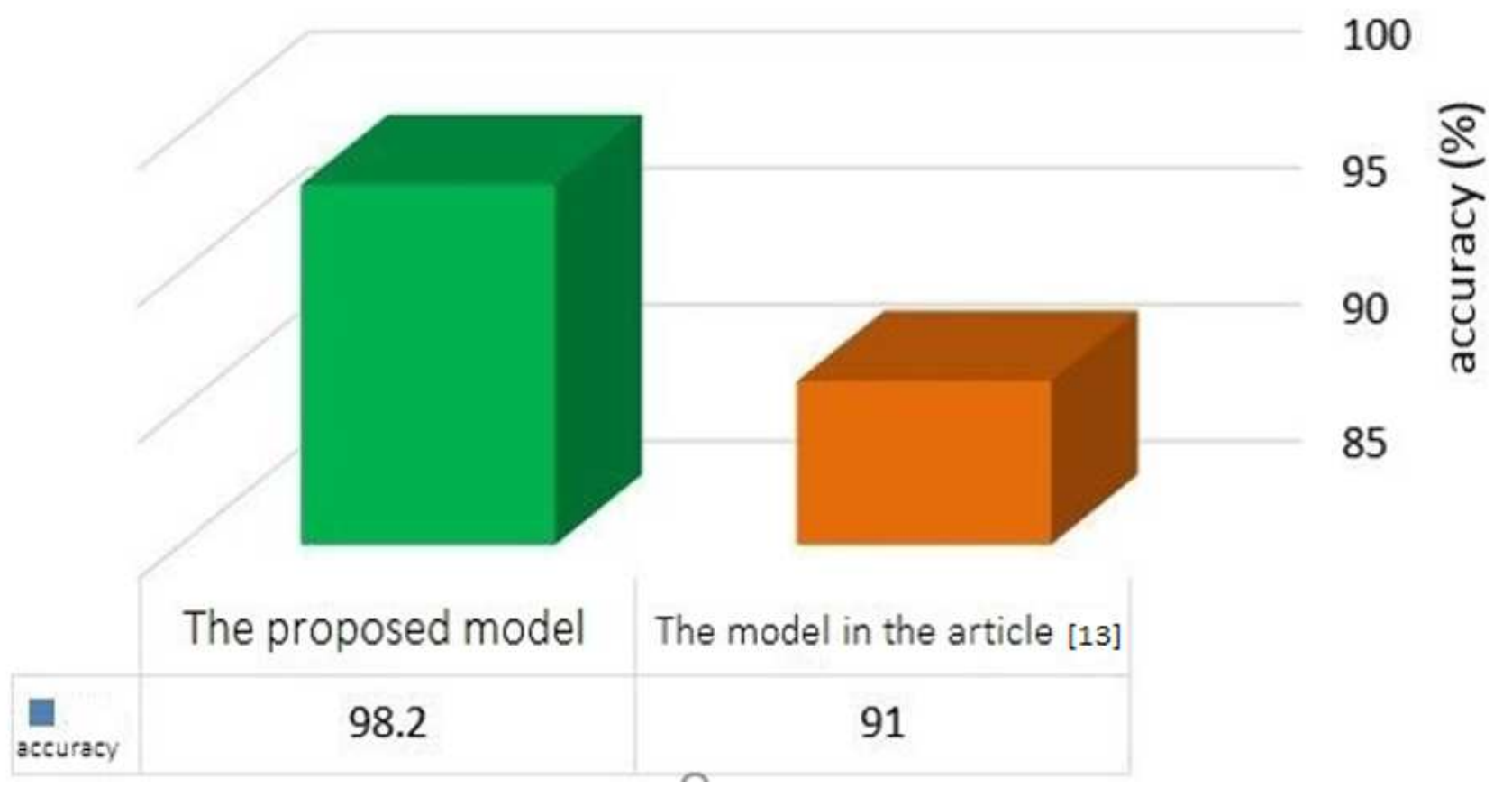

\section{Figure 7}

Comparison of classification accuracy and diagnosis accuracy of the proposed model with those of reference [13] 